\title{
Larvas de linhagens de tilápia do Nilo submetidas à frequências alimentares sob baixa temperatura
}

\author{
Larvae of Nile tilapia lines subject to feeding frequencies under low temperature
}

\author{
SILVA, Emília Tatiane Lopes da ${ }^{1}$; PEDREIRA, Marcelo Mattos ${ }^{1 *}$; DIAS, Maria Letícia \\ Fernandes $^{1}$; TESSITORE, Antônio Jessey de Abreu ${ }^{2}$; FERREIRA, Talita Andrade ${ }^{1}$
}

\footnotetext{
${ }^{1}$ Universidade Federal dos Vales Jequitinhonha e Mucuri, Laboratório de Aquicultura e Ecologia Aquática, Departamento de Zootecnia, Alto da Jacuba, Diamantina, Minas Gerais, Brasil.

${ }^{2}$ Companhia de Desenvolvimento dos Vales do São Francisco e Parnaiba, Morada Nova, Minas Gerais, Brasil.

*Endereço para correspondência: marcelomattospedreira@gmail.com
}

\section{RESUMO}

O manejo alimentar adequado associado a animais com maior rendimento e resistência a ambientes específicos, como águas com temperaturas abaixo da adequada à espécie, são determinantes para uma maior produtividade. Portanto, objetivou-se com o presente estudo comparar o desempenho da larvicultura de duas linhagens de tilápia do Nilo (Oreochromis niloticus) submetidas a diferentes frequências alimentares com temperatura abaixo da faixa ótima para crescimento. Larvas das linhagens tailandesa (720 indivíduos com peso de $24,3 \mathrm{mg}$ e comprimento total de $12,4 \mathrm{~mm}$ ) e GIFT ( 720 exemplares com $22,5 \mathrm{mg}$ e $12,0 \mathrm{~mm}$ ), foram distribuídas em 24 aquários de $4 \mathrm{~L}$, na densidade de 15 larvas. $L^{-1}, 60$ larvas aquário $^{-1}$, sob aeração constante, fotoperíodo natural e temperatura a $23{ }^{\circ} \mathrm{C}$, em sistema com recirculação com termostato. Elas foram submetidas a três frequências alimentares: 2,5 e 8 vezes.dia ${ }^{-1}$, em delineamento inteiramente casualizado, em esquema fatorial 2 linhagens x 3 frequências, com quatro repetições cada. Exceto a temperatura, os demais parâmetros de qualidade da água, amônia, nitrito, nitrato, turbidez, condutividade e $\mathrm{pH}$, mantiveram-se adequados para a espécie. A linhagem tailandesa apresentou maiores comprimentos total e padrão, peso, fator de condição de Fulton, sobrevivência e biomassa, do que a GIFT. A frequência alimentar 5 vezes.dia ${ }^{-1}$ gerou maiores valores para as larvas em peso, comprimento total e padrão, do que as submetidas à frequência de 2 vezes.dia ${ }^{-1}$, e valores iguais, quando comparadas as submetidas à frequência de 8 vezes.dia ${ }^{-1}$. Na temperatura de $23{ }^{\circ} \mathrm{C}$ devese empregar as tilápias da linhagem tailandesa alimentadas 5 vezes.dia ${ }^{-1}$.

Palavras-chave: arraçoamento, GIFT, melhoramento genético, Oreochromis niloticus, tailandesa

\section{SUMMARY}

The adequate food management associated with animals with higher yields and resistance to specific environments, such as waters with temperatures below the appropriate temperature, are determinant for a higher productivity. Therefore, the aim of this study was to compare larviculture performance of two strains of Nile tilapia (Oreochromis niloticus) submitted to different feeding frequencies with temperature below the optimum range for growth. Larvae of Thai (720 individuals with weight of $24.3 \mathrm{mg}$ and total length of $12.4 \mathrm{~mm}$ ) and GIFT strains (720 individuals with weight of $22.5 \mathrm{mg}$ and total length of $12.0 \mathrm{~mm}$ ) were distributed into 24 aquariuns-4L, the density of 15 larvae. $\mathrm{L}^{-1}, 60$ larvae aquarium ${ }^{-1}$, under constant aeration, natural photoperiod and temperature of $23{ }^{\circ} \mathrm{C}$, with recirculation and thermostat. The fish were subjected to three feeding frequencies: 2,5 and 8 times.day $^{-1}$ in a randomized design in a 2 strains $\times 3$ frequencies factorial scheme, with four replicates each. Except the temperature the other parameters of water quality, ammonia, nitrite, nitrate, turbidity, conductivity and $\mathrm{pH}$, remained adequate for the species. The Thai 
lineage showed higher total and standard length, weight, Fulton condition factor, survival and biomass, than GIFT. The feeding frequency 5 times.day ${ }^{-1}$ led to higher values larvae in weight, standard and total length in relation the frequency of 2 times.day $^{-1}$ but, when compared values of frequency 8 times.day $^{-1}$ was not difference. In the temperature $23{ }^{\circ} \mathrm{C}$, Thai strain fed 5 times.day $^{-1}$ should be used.

Keywords: feeding, GIFT, genetic improvement, Oreochromis niloticus, Thai

\section{INTRODUÇÃO}

A tilápia do Nilo (Oreochromis niloticus) é uma espécie ectotérmica, por isso a temperatura do meio onde vive influencia no seu metabolismo físiológico e por consequência no seu desempenho (MOURA et al., 2007). Mesmo podendo tolerar baixas temperaturas, em temperaturas menores que $20^{\circ} \mathrm{C}$ os peixes assimilam a energia exigida apenas para manutenção (LEONARDO et al., 2011).

O sistema de produção intensivo de tilápias no Brasil tem exigido uma constante busca de linhagens mais produtivas. A linhagem tailandesa, nome dado devido ao país de origem, conhecida também como chitralada, foi introduzida no Brasil em 1996, após intensa seleção iniciada ao final dos anos 60. Como resultado dessa seleção, a tilápia tailandesa superou em produtividade a tilápia do Nilo de origem (PINHEIRO et al., 2006).

A linhagem GIFT (Genetically Improved Farming Tilapia) desenvolvida inicialmente pelo International Center for Living Aquatic Resources Management (ICLARM) - atual World Fish Center, foi introduzida no Brasil em 2005 pela Universidade Estadual de Maringá, PR (FÜLBER et al., 2009). Essa linhagem surgiu do cruzamento de oito linhagens, sendo quatro africanas selvagens (capturadas em Gana, Egito, Quênia e Senegal) e quatro linhagens domesticadas na Ásia (produzidas nas Filipinas, Israel, Singapura, Taiwan e Tailândia) (MASSAGO et al., 2010). Desta forma, a Tilápia GIFT também apresentou índices zootécnicos superiores às tilápias que lhe deram origem.

A frequência de arraçoamento adequada é determinante no manejo aquícola, pois esta se traduz em melhor rendimento do animal e qualidade de água, além de menor custo e desperdício (CARNEIRO \& MIKOS, 2005). Quanto menos desenvolvido for o estágio do indivíduo, maior é a sua atividade metabólica e necessidade de frequência alimentar (FOLKVORD \& OTTERA, 1993). Por outro lado, quando a dieta é dividida em várias refeições diárias, normalmente observa-se uma melhora na digestibilidade, além de redução na excreção (JOHNSTON et al., 2003).

Portanto, objetivou-se com o presente estudo comparar o desempenho da larvicultura das linhagens de tilápias tailandesa e GIFT submetidas à frequências alimentares sob baixa temperatura, a fim de avaliar a possibilidade de se prolongar o período de produção de alevinos, em épocas mais frias, adentrando o inverno.

\section{MATERIAL E MÉTODOS}

O experimento foi conduzido na Universidade Federal dos Vales do Jequitinhonha e Mucuri durante 28 dias em janeiro de 2015, com larvas provenientes da CODEVASF de Nova Porteirinha - MG. Larvas irmãs das linhagens tailandesa (720 indivíduos) e GIFT (720 indivíduos), com seus respectivos pesos $(24,3 \pm 2,41 ; 22,5 \pm$ $2,97 \mathrm{mg})$ e comprimentos totais $(12,4 \pm$ 
0,$93 ; 12,0 \pm 0,89 \mathrm{~mm}$ ), similares entre si $(\mathrm{p}<0,05)$, foram contadas individualmente e transferidas para 24 aquários com volume útil de $4 \mathrm{~L}$, na densidade de 15 larvas.L $L^{-1}, \quad 60$ indivíduos.aquário ${ }^{-1}$, mantidas sob aeração constante e fotoperíodo natural.

As larvas foram submetidas à três frequências alimentares: 2 vezes.dia ${ }^{-1}$ $(7: 30 \mathrm{~h}$ e $18: 00 \mathrm{~h}), 5$ vezes.dia ${ }^{-1}(7: 30 \mathrm{~h}$, 10:30 h, 12:00 h, 15:00 h e 18:00 h) e 8 vezes.dia ${ }^{-1}(7: 30 \mathrm{~h}$, 9:00 h, 10:30 h, 12:00 h, 13:30 h, 15:00 h, 16:30 h e 18:00 h), sob temperatura média de $23 \pm 1{ }^{\circ} \mathrm{C}$, mantida através de termostato em sistema de recirculação. $\mathrm{O}$ experimento foi realizado em um delineamento inteiramente casualizado, num esquema fatorial 2 linhagens x 3 frequências, com quatro repetições cada.

A quantidade de alimento fornecido diariamente foi $20 \%$ da biomassa média inicial de ambas as linhagens $(296,4 \mathrm{mg}$ para tailandesa e $267,6 \mathrm{mg}$ para GIFT). Utilizou-se uma ração comercial em pó com umidade (máx.) 10\%, proteína bruta (mín.) $50 \%$, extrato etéreo (mín.) 4\%, matéria fibrosa (máx.) $6 \%$, matéria mineral (máx.) $18 \%$, cálcio (máx.) $5 \%$, fósforo (mín.) $0,15 \%$, segundo especificações do fabricante, acrescida de $60 \mathrm{mg}$ de $17 \alpha$-metiltestosterona. $\mathrm{kg}^{-1}$ de ração, para reversão sexual, segundo Zanardi et al. (2011).

Diariamente, às $7 \mathrm{~h}$ foram monitorados a temperatura da água $\left({ }^{\circ} \mathrm{C}\right)$, o oxigênio dissolvido (mg. $\left.\mathrm{L}^{-1}\right), \quad$ o $\quad \mathrm{pH} \quad \mathrm{e} \quad \mathrm{a}$ condutividade $\left(\mu \mathrm{S} . \mathrm{cm}^{-1}\right)$ com sonda YSI Profissional Plus. Após a leitura das variáveis limnológicas foi realizado o sifonamento para a limpeza e renovação de água dos aquários, no período da manhã $(50 \%)$ e no final da tarde (50\%), totalizando $100 \%$ de renovação do volume.dia ${ }^{-1}$.

Semanalmente os animais foram pesados para ajuste da quantidade de ração fornecida. Também foram coletadas, no período da manhã $(7 \mathrm{~h})$, antes do sifonamento dos aquários, amostras de água para análises da amônia, nitrito e nitrato, sendo realizadas conforme métodos especificados em "Standard Methods for the Examination of Waterand Wastewater", editado pela "American Public Health Association" (APHA, 2012), além de ter sido mensurada a turbidez (NTU), com sonda YSI Profissional Plus.

Aos 28 dias, após pesagem e contagem de todos os indivíduos, de cada um dos aquários, observou-se a biomassa e sobrevivência, sendo esta última transformada em arcoseno para análise, mas apresentada em porcentagem. Em seguida, 20 larvas de cada repetição passaram por eutanásia com solução de eugenol, segundo Vidal et al. (2008) e fixadas em solução com formol a 10\% para posterior biometria (peso e comprimentos total e padrão), obtidos por balança analítica Bel Mark M254AI (precisão 0,0001 g) e paquímetro digital EDA (precisão $0,01 \mathrm{~mm}$ ) respectivamente. Posteriormente foi calculado $\mathrm{o}$ fator de condição de Fulton pela expressão: $\mathrm{K}=$ pesox100.comprimento padrão ${ }^{-3}$

A todos os parâmetros avaliados aplicou-se a ANOVA e teste Tukey, em nível de $5 \%$ de probabilidade, no programa estatístico SAS 9.1.

\section{RESULTADOS E DISCUSSÃO}

Não houve interação entre os fatores linhagem e frequência alimentar. As larvas da linhagem tailandesa apresentaram melhor desempenho do que as da linhagem GIFT para os parâmetros: comprimento total, comprimento padrão, ganho de peso, fator de condição de Fulton, sobrevivência e biomassa (Tabela 1). 
Isso provavelmente foi devido a interação da capacidade genética com a condição de cultivo. A linhagem tailandesa tem demonstrado bons rendimentos diante de outras linhagens. Quando comparada com a linhagem vermelha, a tailandesa possui maior ganho de peso (CARMO et al., 2008), comprimento, peso final, fator de condição de Fulton e biomassa (MAINARDES - PINTO et al., 2011). A tailandesa ainda apresenta maior ganho de peso em relação à nilótica (CARMO et al., 2008; TENÓRIO et al., 2012), porém, seu ganho de peso é similar ao da linhagem mestiça (TENÓRIO et al., 2012). Devido aos bons resultados de desempenho em diferentes sistemas de cultivo e regiões do país, a linhagem tailandesa vem sendo amplamente cultivada no Brasil (CARMO et al., 2008; MAINARDES PINTO et al., 2011; TENÓRIO et al., 2012).

Tabela 1. Valores médios e desvios-padrão de desempenho das larvas de duas linhagens de tilápias, cultivadas por 28 dias: comprimento total (CT), comprimento padrão $(\mathrm{CP})$, peso, fator de condição de Fulton (K), sobrevivência e biomassa

\begin{tabular}{lccc}
\hline Linhagens & $\mathrm{CT}(\mathrm{mm})$ & $\mathrm{CP}(\mathrm{mm})$ & Peso $(\mathrm{mg})$ \\
\hline Tailandesa & $19,12 \pm 2,67^{\mathrm{a}}$ & $15,05 \pm 2,04^{\mathrm{a}}$ & $142,21 \pm 59,90^{\mathrm{a}}$ \\
GIFT & $17,93 \pm 2,87^{\mathrm{b}}$ & $14,19 \pm 2,25^{\mathrm{b}}$ & $116,27 \pm 67,10^{\mathrm{b}}$ \\
\hline Linhagens & $\mathrm{K}$ & Sobrevivência $(\%)$ & Biomassa $(\mathrm{g})$ \\
\hline Tailandesa & $3,99 \pm 0,16^{\mathrm{a}}$ & $55,70 \pm 5,80^{\mathrm{a}}$ & $5,20 \pm 0,85^{\mathrm{a}}$ \\
GIFT & $3,73 \pm 0,13^{\mathrm{b}}$ & $41,40 \pm 9,92^{\mathrm{b}}$ & $3,43 \pm 0,56^{\mathrm{b}}$ \\
\hline
\end{tabular}

Médias seguidas por letras distintas na mesma coluna diferem pelo Teste de Tukey $(\mathrm{P}<0,05)$.

No presente trabalho, as larvas de tilápias tailandesas apresentaram melhor desempenho quando comparadas com a GIFT, o que pode estar associado a uma maior resistência e adaptação a temperaturas mais baixas. Em trabalho similar Sifa et al. (2002) submetendo as linhagens GIFT, Sudão 78 e Egito 88 a baixas temperaturas, verificou que dentre as três a linhagens a GIFT foi a menos tolerante e que as outras duas não apresentaram diferença entre si. Como principal explicação para essa resposta os autores destacam que a GIFT foi geneticamente melhorada em regiões mais quentes e também que teria sido introduzida na China a menos tempo que as demais linhagens. Coencidência ou não a linhagem chitralada foi introduzida no Brasil em 1996 (PINHEIRO et al., 2006), enquanto que a GIFT foi trazida em 2005 (FÜLBER et al., 2009), tendo um menor período para adaptação as condições ambientais e de cultivo no Brasil. No entanto, verifica-se a queda no consumo de ração resultando em pequeno crescimento quando submetidas à baixas temperaturas, tanto para exemplares da linhagem tailandesa $\left(20{ }^{\circ} \mathrm{C}\right)$ (MOURA et al., 2007) como para as da linhagem GIFT $\left(22{ }^{\circ} \mathrm{C}\right)$ (SANTOS et al., 2013).

Assim como neste trabalho, a preocupação com a seleção de linhagens de tilápias mais tolerantes a baixas temperaturas também vem sendo relatada na China, onde a indústria de tilápia é restrita a uma menor extensão do terrritório daquele país devido as temperaturas inferiores, sendo $\mathrm{o}$ aumento da tolerância da tilápia do Nilo 
à baixas temperaturas uma importante adaptação para prolongar o seu período de crescimento, reduzir a mortalidade e diminuir os custos de produção (SIFA et al., 2002).

Além da diferença de capacidade de suportar baixas temperaturas, o sistema de cultivo ao qual as tilápias estão submetidos pode ser um fator determinante para que a diferença entre as linhagens se manifeste ou não. Fülber et al. (2009) comparando tilápias das linhagens chitralada (tailandesa), GIFT e Bouaké dos 31 aos 58 dias e dos 59 aos 112 dias de idade, submetidas a sistema intensivo, com três densidades de estocagem, para cada faixa de idade, observaram que as linhagens não diferiram quanto aos potenciais de desempenho; comprimento total, altura, peso, ganho de peso e taxa de sobrevivência. Silva et al. (2016) comparando o crescimento de tilápias das linhagens tailandesa e GIFT, em sistema intensivo, sob três densidades de estocagem $(6,25,12,50,18,75$ e 25,00 larva. $\mathrm{L}^{-1}$ ), também observaram que as linhagens não diferiram quanto ao peso, ganho de peso, comprimento padrão, fator de condição de Fulton e sobrevivência. Para Fülber et al. (2009) estes resultados demonstram que 0 potencial genético da linhagem não é o único fator determinante do sucesso na produção, e que essas linhagens devem ser melhor estudadas em sistemas intensivos.

As frequências alimentares 5 e 8 vezes.dia ${ }^{-1}$ proporcionaram larvas com valores de comprimento total, comprimento padrão e peso iguais entre si e maiores do que a frequência 2 vezes.dia ${ }^{-1}$ (Tabela 2). Os valores de fator de condição de Fulton, sobrevivência e biomassa não diferiram entre as frequências alimentares.

Tabela 2. Valores médios e desvios-padrão de desempenho das larvas de duas linhagens de tilápias, submetidas à diferentes frequências alimentares por 28 dias: comprimento total (CT), comprimento padrão (CP), peso, fator de condição de Fulton (K), sobrevivência e biomassa

\begin{tabular}{lccc}
\hline Frequência alimentar & $\mathrm{CT}(\mathrm{mm})$ & $\mathrm{CP}(\mathrm{mm})$ & Peso $(\mathrm{mg})$ \\
\hline 2 vezes.dia $^{-1}$ & $17,61 \pm 2,33^{\mathrm{b}}$ & $13,92 \pm 1,87^{\mathrm{b}}$ & $112,07 \pm 52,37^{\mathrm{b}}$ \\
5 vezes.dia $^{-1}$ & $19,01 \pm 2,80^{\mathrm{a}}$ & $14,99 \pm 2,12^{\mathrm{a}}$ & $135,67 \pm 62,93^{\mathrm{a}}$ \\
8 vezes.dia $^{-1}$ & $19,12 \pm 3,07^{\mathrm{a}}$ & $15,06 \pm 2,36^{\mathrm{a}}$ & $143,41 \pm 73,28^{\mathrm{a}}$ \\
\hline Frequência alimentar $^{\mathrm{a}}$ & $\mathrm{K}$ & Sobrevivência $(\%)$ & Biomassa $(\mathrm{g})$ \\
\hline $\mathbf{2}$ vezes.dia $^{-1}$ & $3,86 \pm 0,22^{\mathrm{a}}$ & $53,1 \pm 10,3^{\mathrm{a}}$ & $4,27 \pm 1,00^{\mathrm{a}}$ \\
8 vezes.dia $^{-1}$ & $3,79 \pm 0,16^{\mathrm{a}}$ & $45,8 \pm 9,8^{\mathrm{a}}$ & $4,11 \pm 1,25^{\mathrm{a}}$ \\
\hline vezes.dia $^{-1}$ & $3,86 \pm 0,13^{\mathrm{a}}$ & $46,7 \pm 12,3^{\mathrm{a}}$ & $4,57 \pm 1,25^{\mathrm{a}}$ \\
\hline
\end{tabular}

Médias seguidas por letras distintas na mesma coluna diferem pelo Teste de Tukey $(\mathrm{P}<0,05)$.

Resultados semelhantes para comprimento e peso com o aumento da frequência alimentar também foram observados para tilápia do Nilo na fase larval, período de reversão sexual, por Sanches \& Hayashy (2001), alimentando as tilápias de 2 a 6 vezes.dia ${ }^{-1}$, com temperatura média as $8 \mathrm{~h}$ de $24,89{ }^{\circ} \mathrm{C}$ e por Meurer et al. (2012), alimentando as tilápais de 1 a 5 vezes.dia ${ }^{-1}$, com temperatura média pela manhã de 28,26 ${ }^{\circ} \mathrm{C}$. Porém, resultados diferentes foram observados por Lazzari et al. (2004), cultivando larva de jundiá, $R$. quelen.

$\mathrm{O}$ incremento de ganho em peso com o aumento da frequência alimentar, até 
um determinado limite, parece ser um padrão para espécies de diferentes hábitos e habitats, como se verifica para as espécies como a tilápia do Nilo (SANCHES \& HAYASHY, 2001; RICHE et al., 2004) e jundiá, $R$. quelen (CANTON et al, 2007). No entanto, Van Der Meer et al. (1997), descrevem que a maior frequência alimentar gera pior conversão alimentar, porém, atende de maneira mais eficiente a alta taxa de crescimento, reduzindo a competição e o coeficiente de variação do peso. Esse aumento de conversão poderia ser explicado pelo excesso de frequência alimentar que leva a uma sobrecarga gástrica, reduzindo a eficiência de absorção do alimento. Riche et al. (2004) verificam esse fenômeno para tilápia do Nilo mantida a $28{ }^{\circ} \mathrm{C}$, em que o retorno do apetite após uma refeição de saciedade era de aproximadamente 4 h. Os autores verificaram que os menores (2-3 horas) que o necessário para a retomada do apetite, apresentam sobrecarga gástrica e os alimentados em intervalos de 4-5 horas consumiam tanto quanto evacuavam, resultando em um aumento da eficiência da produção.

Resultados diferentes dos obtidos neste experimento foram descritos por Carneiro \& Mikos (2005), que não observaram diferença no peso de juvenis de jundiá, $R$. quelen alimentados $1,2,3$ e 4 vezes.dia $^{-1}$. Lazzari et al. (2004), cultivando larva de jundiá, $R$. quelen, também não verificaram diferença no peso, entretanto, os animais foram submetidos à altas frequências alimentares, com intervalo entre as ofertas de alimento de 1 e $2 \mathrm{~h}$, períodos muito curtos para que fosse verificada diferenças significativas. Similarmente, larvas de tilápia quando submetidas a menores intervalos entre refeições, frequências acima de 3 vezes.dia ${ }^{-1}$, não apresentam diferença no crescimento

(SANCHES \&
HAYASHY, 2001; MEURER et al., 2012).

A frequência alimentar não alterou os valores de sobrevivência das larvas para ambas as linhagens. Resultados semelhantes também foram observados para tilápia do Nilo na fase larval por Meurer et al. (2012) e juvenis por Riche et al. (2004). O aumento da frequência alimentar também não alterou a taxa de sobrevivência de outras espécies como pacu, Piaractus mesopotamicus (DIETERICH et al., 2013), jundiá, $R$. quelen (LAZZARI et al., 2004) e para o tricogaster, Trichogaster trichopterus (ZUANON et al., 2004). Contudo, outros resultados são possíveis, como verificouse para jundiá, $R$. quelen, alimentados 1 , 2 e 4 vezes.dia ${ }^{-1}$ (CANTON et al., 2007) e peixe-rei, Odontesthes humensis submetidos as frequências alimentares de 1, 3, 6 e 8 vezes.dia ${ }^{-1}$ (POUEY et al., 2012), em que a sobrevivência foi menor quando os peixes foram alimentados 1 vez.dia ${ }^{-1}$. Possivelmente, a menor sobrevivência para a menor frequência alimentar justifica-se devido ao aumento de competição por alimento e menor conversão e consumo, como sugerido por Van Der Meer et al. (1997).

Os valores de fator de condição de Fulton e biomassa não diferiram em função das frequências alimentares. Lazzari et al. (2004), também não encontraram diferença para o fator de condição de Fulton em peixes submetidos à diferentes frequências alimentares. Para carpa comum, Cyprinus carpio, Bittencourt et al. (2013), também não verificaram diferença para fator de condição de Fulton submetidas às frequências de $1 \mathrm{e}$ 4 vezes dia $^{-1}$.

As frequências alimentares também não proporcionaram diferença na biomassa para juvenis de piava, Leporinu sobtusidens (COPATTI et al., 2013). Já Zuanon et al. (2004), submetendo 
tricogaster, $T$. trichopterus à três frequências alimentares, verificaram maior fator de condição de Fulton e biomassa para indivíduos alimentados 9 vezes.dia ${ }^{-1}$ quando comparados aos alimentados 3 e 6 vezes.dia ${ }^{-1}$. Sendo assim, o efeito da frequência alimentar no crescimento do peixe pode variar de acordo com a espécie, fase de desenvolvimento dentre outras condições.

Quanto às variáveis limnológicas foi possível observar que a amônia, condutividade e $\mathrm{pH}$, não variaram significativamente, entre as frequências alimentares 2, 5 e 8 vezes.dia ${ }^{-1}$ (Tabela 3). Já turbidez aumentou de acordo com o aumento da frequência alimentar. $\mathrm{O}$ contrário foi observado para o nitrito que apresentou maior índice para menor frequência alimentar, diferindo entre as frequências de 2 e 8 vezes.dia ${ }^{-1}$. Já para o nitrato não variou com a frequência alimentar.

Tabela 3. Valores médios e desvios-padrão dos parâmetros de qualidade da água dos aquários para as três frequências alimentares avaliadas para as larvas de tilápia, para o $18^{\circ}$ dia de experimento

\begin{tabular}{|c|c|c|c|}
\hline \multirow{2}{*}{ Parâmetros da água } & \multicolumn{3}{|c|}{ Frequências alimentares } \\
\hline & 2 vezes.dia ${ }^{-1}$ & 5 vezes.dia ${ }^{-1}$ & 8 vezes.dia $^{-1}$ \\
\hline Amônia não ionizada $\left(\mathrm{mg} \cdot \mathrm{L}^{-1}\right)$ & $0,01 \pm 0,004^{\mathrm{a}}$ & $0,01 \pm 0,003^{\mathrm{a}}$ & $0,01 \pm 0,003^{\mathrm{a}}$ \\
\hline Nitrito $\left(\mathrm{mg} \cdot \mathrm{L}^{-1}\right)$ & $0,53 \pm 0,15^{\mathrm{a}}$ & $0,43 \pm 0,19^{\mathrm{ab}}$ & $0,31 \pm 0,06^{\mathrm{b}}$ \\
\hline Nitrato $\left(\mathrm{mg} \cdot \mathrm{L}^{-1}\right)$ & $2,51 \pm 1,21^{\mathrm{a}}$ & $1,10 \pm 0,48^{\mathrm{a}}$ & $0,96 \pm 1,33^{\mathrm{a}}$ \\
\hline Turbidez (NTU) & $4,55 \pm 2,12^{\mathrm{c}}$ & $7,15 \pm 1,78^{\mathrm{b}}$ & $12,25 \pm 2,91^{\mathrm{a}}$ \\
\hline Condutividade $\left(\mu \mathrm{S} . \mathrm{cm}^{-1}\right)$ & $82,65 \pm 14,69^{\mathrm{a}}$ & $81,80 \pm 11,35^{\mathrm{a}}$ & $99,70 \pm 18,25^{\mathrm{a}}$ \\
\hline pH & $6,02 \pm 0,08^{\mathrm{a}}$ & $6,08 \pm 0,13^{\mathrm{a}}$ & $6,22 \pm 0,19^{\mathrm{a}}$ \\
\hline
\end{tabular}

Médias seguidas por letras distintas na mesma linha diferem pelo Teste de Tukey $(\mathrm{P}<0,05)$.

Em sistemas de criação, o alimento, o adubo, o fertilizante e as fezes dos peixes são fontes de nitrogênio (MERCANTE et al, 2007). Os valores médios das concentrações de amônia não ionizada para as frequências: $2,5 \mathrm{e}$ 8 vezes.dia $^{-1}$, foram adequados para o cultivo de tilápia, já que estiveram abaixo de $0,08 \mathrm{mg} . \mathrm{L}^{-1}$, concentração em que começam seu efeito tóxico com a perda de apetite pelos animais (POPMA \& LOVSHIN, 1996).

Quando comparados os parâmetros limnológicos paras as linhagens tailandesa e GIFT, observou-se que o nitrito, condutividade e $\mathrm{pH}$ não apresentaram variações significativas entre as mesmas. Já a amônia apresentou valores maiores para tailandesa com relação aos valores apresentados para a GIFT, assim como a turbidez. Os valores de nitrato foram maiores para GIFT (Tabela 4).

Assim como observado entre as frequências alimentares os valores médios das concentrações de amônia não ionizada, estiveram dentro dos limites considerados por Popma \& Lovshin (1996), como adequados para o cultivo de tilápia. A linhagem tailandesa apresentou maior crescimento e assim, maior demanda de alimento o que levou uma maior concentração de amônia e maior turbidez da água quando comparada com a GIFT. 
Tabela 4. Valores médios e desvios-padrão dos parâmetros de qualidade da água dos aquários experimentais para as duas linhagens de larvas de tilápias avaliadas, para o $18^{\circ}$ dia de experimento

\begin{tabular}{lcc}
\hline \multirow{2}{*}{ Parâmetros da água } & \multicolumn{2}{c}{ Linhagens } \\
\cline { 2 - 3 } & $0,011 \pm 0,002^{\mathrm{a}}$ & Gailandesa \\
\hline Amônia não ionizada $\left(\mathrm{mg} . \mathrm{L}^{-1}\right)$ & $0,49 \pm 0,20^{\mathrm{a}}$ & $0,004 \pm 0,003^{\mathrm{b}}$ \\
Nitrito (mg. $\left.\mathrm{L}^{-1}\right)$ & $1,25 \pm 0,51^{\mathrm{b}}$ & $2,38 \pm 0,08^{\mathrm{a}}$ \\
Nitrato $\left(\mathrm{mg} . \mathrm{L}^{-1}\right)$ & $10,20 \pm 3,25^{\mathrm{a}}$ & $6,15 \pm 2,17^{\mathrm{a}}$ \\
Turbidez (NTU) & $93,20 \pm 17,34^{\mathrm{a}}$ & $79,85 \pm 12,02^{\mathrm{a}}$ \\
Condutividade $\left(\mu \mathrm{S} . \mathrm{cm}^{-1}\right)$ & $6,05 \pm 0,15^{\mathrm{a}}$ & $6,05 \pm 0,13^{\mathrm{a}}$ \\
$\mathrm{pH}$ &
\end{tabular}

Médias seguidas por letras distintas na mesma linha diferem pelo Teste de Tukey $(\mathrm{P}<0,05)$.

Já o nitrito teve suas concentrações para os diferentes tratamentos e linhagens abaixo dos limites $\left(0,7\right.$ e $\left.1 \mathrm{mg} . \mathrm{L}^{-1}\right)$ considerados como adequados para o cultivo de tilápia do Nilo (MERCANTE et al., 2007). Portanto, os níveis de nitrito obtidos no presente estudo, muito provavelmente não afetaram negativamente as larvas.

A concentração de nitrato foi maior para a linhagem GIFT, que, associado à maior concentração de amônia para a linhagem tailandesa, permite concluir que $\mathrm{o}$ ambiente de cultivo da tailandesa ficou mais saturado. No entanto, os valores observados na criação das duas linhagens de tilápia estiveram bem abaixo do limite superior da faixa adequada (MERCANTE et al., 2007).

A turbidez está relacionada com aporte de matéria orgânica proveniente do arraçoamento (MERCANTE et al., 2007). Assim sendo, a maior turbidez observada para as larvas de tailandesa pode ser reflexo da maior quantidade de matéria orgânica no meio devido ao seu melhor desempenho em relação às GIFT. Altos níveis de turbidez podem prejudicar o sistema de cultivo ao interferir na visualização dos alimentos pelos peixes e no processo de trocas gasosas ao obstruir as brânquias, podendo levar a morte do animal (BALDISSEROTTO, 2009). No entanto, os valores de turbidez observados para este experimento estiveram bem abaixo do valor (100 NTU) considerado por Mercante et al. (2007) como limite máximo da faixa adequada para o cultivo de peixes.

Já a condutividade elétrica manteve-se com valores que segundo Mercante et al. (2007), está acima do limite aceitável e pode ser um indicativo de elevada matéria orgânica em decomposição. No entanto, os valores de condutividade formam similares aos observados pelo próprio Mercante et al. (2007) e por Meurer et al. (2012), para tilápia, onde não são descritos nenhuma mortandade ou comprometimento do crescimento em função deste parâmetro, sugerindo maiores estudos quanto a influência da condutividade.

$\mathrm{O} \mathrm{pH}$ e o oxigênio estiveram dentro dos limites considerados por Mercante et al. (2007), como aceitáveis para a tilápia. Por fim, os parâmetros de qualidade de água estiveram adequados ao cultivo da espécie, mesmo os que apresentaram diferenças significativas entre os tratamentos.

Desse modo, indica-se o emprego das larvas de tilápias da linhagem tailandesa alimentadas 5 vezes.dia ${ }^{-1}$, para temperatura de $23^{\circ} \mathrm{C}$, o que permite prolongar o período de produção de alevinos, adentrando o inverno. 


\section{AGRADECIMENTOS}

À Fundação de Amparo à Pesquisa do Estado de Minas Gerais, pelo apoio financeiro e pela concessão de bolsa, à Coordenação de Aperfeiçoamento de Pessoal de Nivel Superior pela concessão de bolsa e ao Conselho Nacional de Desenvolvimento Científico e Tecnológico e ao Banco do Nordeste do Brasil, pelo apoio financeiro.

\section{REFERÊNCIAS}

AMERICAN PUBLIC HEALTH ASSOCIATION - APHA. Standard methods for examination of water and wastewater. 22nd Washington: Water Environment Federation, 2012. $1.496 \mathrm{p}$.

BALDISSEROTTO, B. Fisiologia de peixes aplicada à piscicultura. 2.ed. Santa Maria: UFSM, 2009. 350p.

\section{BITTENCOURT, F.; DACLEY}

HERTES, N.E.U.; POZZER, R.; LUI, T.A.; FEIDEN, A.; BOSCOLO, W.R. Frequência de arraçoamento para alevinos de carpa comum. Boletim do Instituto de Pesca, v.39, n.2, p.149156, 2013.

CANTON, R.; WEINGARTNER, M.; FRACALOSSI, D.M.; ZANIBONI FILHO, E. Influência da frequência alimentar no desempenho de juvenis de jundiá. Revista Brasileira de

Zootecnia, v.36, n.4, p.749-753, 2007.

CARMO, J.L.; FERREIRA, D.A.; DA SILVA JÚNIOR, R.F.; DE SOUZA SANTOS, R.M.; DE SOUZA CORREIA, E. Crescimento de três linhagens de tilápia sob cultivo semiintensivo em viveiros. Revista Caatinga, v.21, n.2, p.20-26, 2008.
CARNEIRO, F.P.C.; MIKOS, J.D. Frequência alimentar e crescimento de juvenis de jundiá, Rhamdia quelen.

Ciência Rural, v.35, n.1, p.187-191, 2005.

COPATTI, C.E.; SANTOS, T.A.D.; GARCIA, S.F.S. Densidade de estocagem e frequência alimentar de juvenis de piava Leporinus obtusidens valenciennes, 1836 (Characiformes: anostomidae). Current Agricultural Science and Technology, v.14, n.4, p.107-111, 2013.

DIETERICH, T.G.; POTRICH, F.R.; LORENZ, E.K.; SIGNOR, A.A.; FEIDEN, A.; BOSCOLO, W.R.

Parâmetros zootécnicos de juvenis de pacu alimentados a diferentes frequências de arraçoamento em tanques-rede.

Pesquisa Agropecuária Brasileira, v.48, n.8, p.1043-1048, 2013.

FOLKVORD, A.; OTTERA, H. Effects of initial size distribution day length, and feeding frequency on growth, survival, and cannibalism in juvelile Atlantic cod (Gadusmorhua, L.). Aquaculture, v.114, n.3, p.243-260, 1993.

FÜLBER, V.M.; MENDEZ, L.D.V.; BRACCINI, G.L.; BARRERO, N.M.L.; DIGMEYER, M.; RIBEIRO, R.P. Desempenho comparativo de três linhagens de tilápia do Nilo Oreochromis niloticus em diferentes densidades de estocagem. Acta Scientiarum: Animal Sciences, v.31, n.2, p.177-182, 2009.

JOHNSTON, G.; KAISER, H.; HECHT, T.; OELLERMANN, L. Effect of ration size and feeding frequency on growth, size distribution and survival of juvenile clownfish, Amphiprion percula. Journal of Applied Ichthyology, v.19, n.1, p.4043, 2003. 
LAZZARI, R.; RADUNZ NETO, J.; LIMA, R.; PEDRON, F.; LOSEKANN, M. Efeito da freqüência de arraçoamento e da troca do tamanho de partícula alimentar no desenvolvimento de pós-larvas de jundiá (Rhamdia quelen). Revista Brasileira de Agrociência, v.10, n.2, p.231-234, 2004.

LEONARDO, A.F.; CORRÊA, C.F.; BACCARIN, A.E. Qualidade da água de um reservatório submetido à criação de tilápias em tanques-rede, no sul de São Paulo, Brasil. Boletim do Instituto de Pesca, v.37, n.4, p.341-354, 2011.

MAINARDES-PINTO, C.S.R.; VERANI, J.R.; SCORVO FILHO, J.D.; DA SILVA, A.L. Desempenho produtivo da tilápia tailandesa e da tilápia vermelha da Flórida estocadas em diferentes densidades, em tanquesrede instalados em viveiros. Boletim do Instituto de Pesca, v.37, n.3, p.225234, 2011.

MASSAGO, H.; CASTAGNOLLI, N.; MALHEIROS, B.; KOBERSTEIN, T.C.R.D.; SANTOS, M.A.; RIBEIRO, R.P. Crescimento de quatro linhagens de tilápia Orechromis niloticus. Revista Acadêmica Ciências Agrárias e Ambientais, v.8, n.4, p.397-203, 2010.

MERCANTE, C.T.J.; MARTINS, Y.K.; CARMO, C.F.; OSTI, J.S.; MAINARDES-PINTO, C.S.R.; TUCCI, A. Qualidade de água em viveiro de Tilápia do Nilo (Oreochromis niloticus): caracterização diurna de variáveis físicas, químicas e biológicas, São Paulo, Brasil. Bioikos, v.21, n.2, p.79-88, 2007.

MEURER, F.; BOMBARDELLI, R.A.; PAIXÃO, P.S.; SILVA, L.C.R.; SANTOS, L.D. Feeding frequency on growth and male percentage during sexual reversion phase of Nile tilapia.

Revista Brasileira de Saúde e

Produção Animal [online], v.13, n.4, p.1133-1142, 2012.

MOURA, G.S.; OLIVEIRA, M.G.A.; LANNA, E.T.A.; JÚNIOR, A.M.; MACIEL, C.M.R.R. Desempenho e atividade de amilase em tilápias-do-Nilo submetidas a diferentes temperaturas.

Pesquisa Agropecuária Brasileira, v.42, n.11, p.1609-1615, 2007.

PINHEIRO, L.M.S.; MARTINS, R.T.; PINHEIRO, L.A.S.; PINHEIRO, L.E.L. Rendimento industrial de filetagem da tilápia tailandesa (Oreochromis spp.). Arquivo Brasileiro de Medicina Veterinária e Zootecnia, v.58, n.2, p.257-262, 2006.

POPMA, T.J.; LOVSHIN, L.L.

Worldwide prospects for commercial production of tilapia. Alabama: International Center for Aquaculture and Aquatic Environments, 1996. 23p.

POUEY, J.L.O.F.; ROCHA, C.B.; TAVARES, R.A.; PORTELINHA, M.K.; PIEDRAS, S.R.N. Frequência alimentar no crescimento de alevinos de peixe-rei Odontesthes humensis.

Semina: Ciências Agrárias, v.33, n.6, p.2423-2428, 2012.

RICHE, M.; HALEY, D.I.; OETKER, M.; GARBRECHT, S.; GARLING, D.L. Effect of feeding frequency on gastric evacuation and the return of appetite in tilapia Oreochromis niloticus (L.). Aquaculture, v.234, n.1, p.657$673 ; 2004$.

SANCHES, L.E.F.; HAYASHI, C. Effect of feeding freequency on Nile tilapia, Oreochromis niloticus (L.) fries performance during sex reversal in hapas. Acta Scientiarum: Animmal Science, v.23, n.4, p.871-876, 2001. 
SANTOS, B.S.; MARECO, E.A.; SILVA, M.D.P. Growth curves of Nile tilápia (Orechromis niloticus) strains cultivation at different temperatures. Acta Scientiuarium. Animal Science, v.35, n.3, p.235-242, 2013.

SIFA, L.; CHENHONG, L.; DEY, M.; GAGALAC, F.; DUNHAM, R. Cold tolerance of three strains of Nile tilapia, Oreochromis niloticus, in China.

Aquaculture, v.213, n.1-4, p.123-129, 2002.

SILVA, E.T.L.; PEDREIRA, M.M.; MOURA, G.S.; PEREIRA, D.K.A.; OTONI, C.J. Larval culture of nile tilapia lineages at different storage densities. Revista Caatinga, v.29, n.3, p.709-715, 2016.

TENÓRIO, I.V.; SOARES, M.D.C.F.; LOPES, J.P. Desempenho comparativo em tanques-rede de três linhagens da tilápia do Nilo - Oreochromis niloticus: comum, chitralada e mestiço. Revista Biotemas, v.25, n.1, p.65-72, 2012.

VAN DER MEER, M.B.; VAN HERWAARDEN, H.; VERDEGEM, M.C.J. Effect of number of meals and frequency of feeding on voluntary feed intake of Colossomama cropomum (Cuvier). Aquaculture Research, v.28, n.6, p.419-432, 1997.

VIDAL, L.V.O.; ALBINATI, R.C.B.; ALBINATI, A.C.L.; LIRA, A.D.; ALMEIDA, T.R.; SANTOS, G.B. Eugenol como anestésico para a tilápiado-nilo. Pesquisa Agropecuária Brasileira, v.43, n.8, p.1069-1074, 2008.
ZANARDI, M.F.; DIAS-

KOBERSTEIN, T.C.R.; SANTOS, M.A.; MALHEIRO, E.B. Desempenho produtivo e reversão sexual em tilápias em dois métodos hormonal.

Veterinária e Zootecnia, v.18, n.1, p.45-52, 2011.

ZUANON, J.A.S.; ASANO, M.; FERNANDES, J.B.K. Performance of Tricogaster (Trichogaster trichopterus) submitted to different feeding levels and stocking densities. Revista Brasileira de Zootecnia, v.33, n.6, p.1639-1645, 2004.

Data de recebimento: 01/05/2016

Data de aprovação: 24/10/2016 